\title{
Serum vascular endothelial growth factor-C levels predict lymph node metastasis and prognosis of patients with gallbladder cancer
}

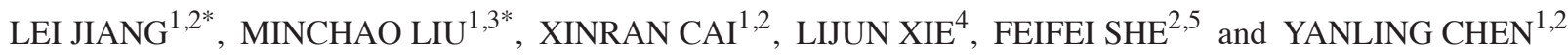 \\ ${ }^{1}$ Department of Hepatobiliary Surgery, Union Clinical Medical College, Fujian Medical University, Fuzhou, Fujian 350001; \\ ${ }^{2}$ Key Laboratory of The Ministry of Education for Gastrointestinal Cancer, Fujian Medical University, Fuzhou, Fujian 350108; \\ ${ }^{3}$ Department of General Surgery, Zhangzhou Affiliated Hospital of Fujian Medical University, Zhangzhou, Fujian 363000; \\ ${ }^{4}$ Department of Ultrasonic Image, The First Clinical Medical College, Fujian Medical University, Fuzhou, Fujian 350005; \\ ${ }^{5}$ Key Laboratory of Tumor Microbiology, School of Basic Medical Sciences, \\ Fujian Medical University, Fuzhou, Fujian 350108, P.R. China
}

Received August 28, 2017; Accepted July 4, 2018

DOI: $10.3892 / \mathrm{ol} .2018 .9358$

\begin{abstract}
Lymph node metastasis is the primary site of metastasis for patients with gallbladder cancer (GBC). Vascular endothelial growth factor-C (VEGF-C) has been implicated in the control of lymphangiogenesis and lymph node metastasis in various malignant tumors. However, the function of circulating VEGF-C is unclear and it is often difficult to evaluate lymph node metastasis and provide a prognosis for GBC. In the present study, ELISA was used to measure the preoperative serum VEGF-C (sVEGF-C) levels of 51 patients with GBC, 15 patients with chronic cholecystitis and 10 healthy volunteers. The results revealed a significantly increased sVEGF-C level in patients with GBC compared with the healthy donors, however no statistically significant difference was identified between patients with GBC and chronic cholecystitis. sVEGF-C levels were associated with lymph node metastasis in GBC and presented a positive correlation with VEGF-C expression and lymphatic vessel density (LVD) in patients with GBC. The mean survival time with high sVEGF-C was significantly reduced compared with low sVEGF-C. A similar result was also observed for VEGF-C expression and LVD. In summary, sVEGF-C levels may predict lymph node metastasis and the prognosis of patients with GBC.
\end{abstract}

Correspondence to: Dr Yanling Chen, Department of Hepatobiliary Surgery, Union Clinical Medical College, Fujian Medical University, 29 Xinquan Road, Gulou, Fuzhou, Fujian 350001, P.R. China

E-mail: drchenyl@126.com

Dr Lijun Xie, Department of Ultrasonic Image, The First Clinical Medical College, Fujian Medical University, 20 Chazhong Road, Taijiang, Fuzhou, Fujian 350005, P.R. China

E-mail: haohaohaojunm@163.com

*Contributed equally

Key words: sVEGF-C, lymph node metastasis, prognosis, gallbladder cancer

\section{Introduction}

Gallbladder cancer (GBC) is a highly malignant tumor, and its pathogenesis remains unknown at present. GBC symptoms remain inconspicuous in the early stages. When patients exhibit remarkable symptoms, metastasis has already advanced. Lymph node metastasis is the primary mode of transfer of GBC; the first step involves the spread of cancer cells to the lymph nodes via lymphatic vessels. The overall rate of lymph node metastasis in GBC ranges from 54 to $64 \%$ (1). Early diagnosis of gallbladder carcinoma presents difficulty, and lymph node metastasis is also early. Therefore, treatment of GBC bears importance for early detection of lymphatic metastasis caused by cytokines in the blood.

Vascular endothelial growth factor-C (VEGF-C), a member of the VEGF family, has been found to induce lymphangiogenesis and promote lymph node metastasis in many malignant tumors (2-4). Karpanen et al (5) observed that VEGF-C facilitates tumor metastasis via the lymphatic vessels, and that tumor spread can be inhibited by blocking the interaction between VEGF-C and its receptors. Kitadai et al (6) suggested that VEGF-C may play a role in tumor progression via lymphangiogenesis and angiogenesis in human esophageal carcinoma. Our previous study (7) also demonstrated the involvement of VEGF-C in lymphangiogenesis and angiogenesis of GBC and promotion of lymph node metastasis in GBC.

However, insufficient information describes the relationship between VEGF-C expression at the circulating level and lymph node metastasis in GBC. In this study, we aimed at characterizing the role of serum VEGF-C (sVEGF-C) in predicting lymph node metastasis and prognosis of patients with GBC. We observed that SVEGF-C level was significantly higher in patients with GBC than in healthy donors. We then measured the positive association between sVEGF-C and expression of VEGF-C in tissue samples of human GBC. We also showed that SVEGF-C level was significantly associated with lymphatic vessel density (LVD) and lymph node metastasis. We demonstrated that mean survival time was significantly shorter with high sVEGF-C than with low sVEGF-C. Altogether, these findings suggest that sVEGF-C 
levels may predict lymph node metastasis and prognosis of patients with GBC.

\section{Patients and methods}

Patients and tissue specimens. The study was performed in 51 patients with histopathologically proven GBC and who underwent potentially curative surgery without preoperative therapy or transfusion in the Affiliated Union Hospital of Fujian Medical University (Fuzhou, China) between 2005 and 2016. The patient group comprised 20 men and 31 women with a median age of 59 years (32-84 years old). All patients were staged clinically according to the American Joint Committee on Cancer (AJCC; 7th edition) (8). According to operative notes and pathological results, 27 and 16 patients presented lymph node metastasis and distant metastasis, respectively. A total of 15 patients with chronic cholecystitis were treated by surgery in the Affiliated Union Hospital of Fujian Medical University, and 10 healthy volunteers were used as controls. Paraffin-embedded specimens were collected according to the protocol approved by the Ethics Committee of the Affiliated Union Hospital of Fujian Medical University.

Blood sVEGF-C level assay with enzyme-linked immunoadsorbent assay (ELISA). Peripheral venous blood samples were collected on the day prior to surgery or anticancer therapy. Blood samples were collected according to the protocol approved by the Ethics Committee of the Affiliated Union Hospital of Fujian Medical University. Serum samples were separated from the blood by centrifugation at 2,000 r/min for $15 \mathrm{~min}$ and kept frozen at $-80^{\circ} \mathrm{C}$ until the assay. Measurement of VEGF-C was performed using the Quantikine Human VEGF-C Immunoassay kit (R\&D Systems, Minneapolis, MN, USA) according to manufacturer's instructions. All assays were duplicated. Sensitivity limit of the method for VEGF-C reached $13.3 \mathrm{pg} / \mathrm{ml}$.

VEGF-C and D2-40 expression with immunohistochemical staining. Human GBC specimens were collected after operation and were fixed in $10 \%$ formaldehyde solution and embedded in paraffin. The paraffin-embedded tissues were cut into $4 \mathrm{~mm}$ serial sections and mounted on glass slides. Tissue sections were deparaffinized with xylene, dehydrated in ethanol, and incubated with $3 \%$ hydrogen peroxidase $\left(\mathrm{H}_{2} \mathrm{O}_{2}\right)$ for $20 \mathrm{~min}$ to block endogenous peroxidase activity. After washing with phosphate-buffered saline (PBS), the tissue sections were antigen-retrieved by heating in a microwave for $13 \mathrm{~min}$ in a citric acid buffer solution ( $\mathrm{pH}$ 6.0). Tissue sections were blocked by $15 \mathrm{~min}$ incubation with $5 \%$ rabbit serum, followed by an overnight incubation with VEGF-C polyclonal goat anti-human antibody (1:80; Santa Cruz Biotechnology, Santa Cruz, CA, USA) and D2-40 monoclonal mouse anti-human antibody (1:100; Maixin-Bio, Fuzhou, China) in humidified boxes at $4^{\circ} \mathrm{C}$. The sections were then washed with PBS for 5 min and incubated with an UltraSensitive S-P kit (Maixin-Bio) according to manufacturer's instructions. After exposure to stable III, 3-diaminobenzidine for 5-10 min, the slides were counterstained with hematoxylin and eosin, dehydrated, and mounted. For the negative control, PBS was used instead of the primary antibody. VEGF-C expression was semiquantitatively evaluated to be negative and positive based on a previously described scoring system (Fig. 1A and B) (9). Average of D2-40-positive was assessed according to the method described by Takammi I (Fig. 1C) (10). Each slide was first scanned at magnification, x100 to determine three 'hot spots', which were defined as areas with the maximum number of positive vessels, and all the immunostained vessels at magnification, $\mathrm{x} 400$ were counted to determine the positive vessel density. The average number of positive vessel in the five selected areas was calculated for each case.

Statistical analysis. Data are expressed as mean \pm standard deviation for continuous variables. Student's t-test was used to analyze continuous variables for two groups and one-way ANOVA for more than two groups. Chi-square test was used to compare categorical variables. Kaplan-Meier survival analysis was used to estimate survival time, and Mantel's long-rank test was used to compare differences in survival time. Statistical analyses were performed using the SPSS software (version 17.0; SPSS Inc., Chicago, IL, USA). P<0.05 was considered to indicate a statistically significant difference.

\section{Results}

Relatively high sVEGF-C levels in GBC patient group. Preoperative sVEGF-C level in patients with GBC totaled $7846.17 \pm 1915.6 \mathrm{pg} / \mathrm{ml}$ (median \pm standard deviation), which was significantly higher than that in healthy volunteers (4828.1 $\pm 1547.12 \mathrm{pg} / \mathrm{ml} ; \mathrm{P}=0.00001)$ (Fig. 2). However, no statistical significance was observed in the preoperative sVEGF-C level in patients with GBC and chronic cholecystitis (7425.33 \pm 1784.76 pg/ml, $\mathrm{P}=0.451$ ) (Fig. 2).

Association among sVEGF-C levels, VEGF-C expression, $L V D$, and clinicopathological characteristics of $G B C$. Table I summarizes the associations among sVEGF-C levels, VEGF-C expression, LVD, and clinicopathological characteristics in patients with GBC. sVEGF-C level was associated with lymph node metastasis $(\mathrm{P}=0.001)$. However, no significant association was observed between sVEGF-C and factors, such as age, sex, tumor size, histological type, histological grade, tumor depth, distant metastasis, and stage. VEGF-C expression in human GBC tissue was significantly correlated with lymph node metastasis $(\mathrm{P}=0.02)$ and tumor size ( $\leq 5$ and $>5 \mathrm{~cm} ; \mathrm{P}=0.04$ ) but showed no correlation with age, sex, histological type, histological grade, tumor depth, distant metastasis, and stage. LVD in human GBC tissue was significantly correlated with lymph node metastasis $(\mathrm{P}=0.02)$ but presented no correlation with age, sex, tumor size, histological type, histological grade, tumor depth, distant metastasis, and stage.

SVEGF-C levels exhibited positive correlation with VEGF-C expression and $L V D$ in $G B C$. We analyzed the relationship between sVEGF-C levels and VEGF-C expression in tumor tissues. As the median of preoperative sVEGF-C level in patients with GBC reached $7846.17 \mathrm{pg} / \mathrm{ml}$, we divided the samples into two groups with a mean value of $7846 \mathrm{pg} / \mathrm{ml}$. Considering the mean value of $7846 \mathrm{pg} / \mathrm{ml}$, we divided the samples into two groups $(>7846, \leq 7846 \mathrm{pg} / \mathrm{ml})$ (Table II). The 
A

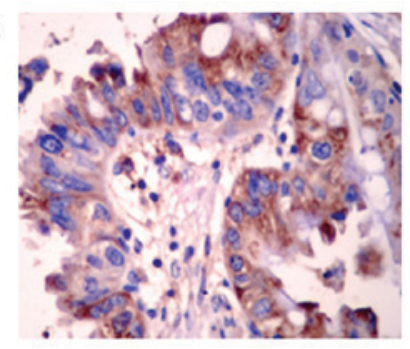

B

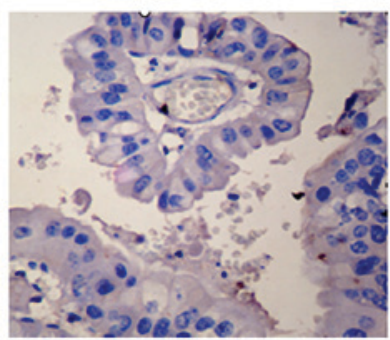

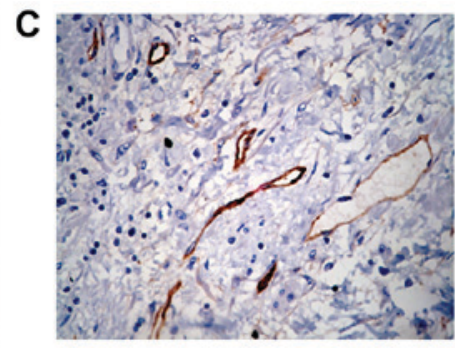

Figure 1. VEGF-C and D2-40 expression in gallbladder cancer. (A) VEGF-C-positive expression. (B) VEGF-C-negative expression. (C) Microlymphatic vessel with D2-40 expression. Magnification, x400. VEGF-C, vascular endothelial growth factor-C.

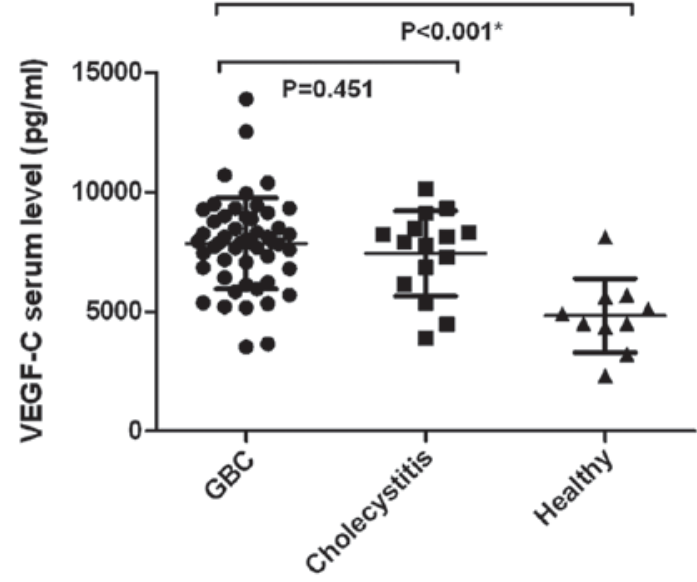

Figure 2. sVEGF-C levels in 51 patients with GBC, 10 healthy controls and 15 patients with chronic cholecystitis. Preoperative sVEGF-C level (median \pm standard deviation) in patients with GBC was significantly higher compared with the healthy volunteers $(7846.17 \pm 1915.6$ vs. $4828 ; 1 \pm 1547$. $2 \mathrm{pg} / \mathrm{ml} ; \mathrm{P}=0.00001)$. No statistical significance was identified in the preoperative sVEGF-C level between patients with GBC and chronic cholecystitis (7425.33 $\pm 1784.76 \mathrm{pg} / \mathrm{ml} ; \mathrm{P}=0.451)$. GBC, gallbladder cancer; sVEGF-C, serum vascular endothelial growth factor-C.

positive rate of VEGF-C expression in human GBC tissue with higher sVEGF-C ( $>7846 \mathrm{pg} / \mathrm{ml})$ group totaled $89.3 \%$, and that in human GBC tissue with lower sVEGF-C ( $\leq 7846 \mathrm{pg} / \mathrm{ml})$ group measured $30.4 \%$. Both differences showed statistical significance at $\mathrm{P}<0.01$. At the same time, a statistically significant decrease in LVD was noted in the lower-sVEGF-C group $(5.0+2.7)$ compared with the higher-sVEGF-C group $(8.5+3.4)(\mathrm{P}<0.01)$.

Correlation among sVEGF-Clevel, VEGF-C expression, $L V D$, and patient survival in $G B C$. In this study, one-year survival rate was $39.2 \%$. Mean survival time of patients with high sVEGF-C levels $(>7846 \mathrm{pg} / \mathrm{ml})$ measured $9.32 \pm 7.18$ months, whereas that of with low sVEGF-C level $(<7846 \mathrm{pg} / \mathrm{ml})$ reached $23.39 \pm 20.40$ months $(\mathrm{P}=0.0118$; Fig. $3 \mathrm{~A})$. As the median of microlymphatic density in patients with GBC was $7 / 400 \mathrm{HP}$, we divided the samples into two groups with mean values of $<7 / 400$ and $>7 / 400$ HP. Mean survival rates of patients in high ( $\geq 7 / 400 \mathrm{HP}$ ) LVD and low ( $<7 / 400 \mathrm{HP})$ LVD groups amounted to $10.08 \pm 6.92$ and $21.48 \pm 20.61$ months, respectively ( $\mathrm{P}=0.0151$; Fig. 3B). Mean survival time was shorter in the VEGF-C-positive expression group than in the negative expression group (11.25 \pm 7.87 vs. $23.11 \pm 22.92$ months; $\mathrm{P}=0.0339$; Fig. 3C).

\section{Discussion}

GBC, a common malignancy with high mortality, refers to the fifth most common cancer among gastrointestinal cancers (11) and accounts for $80-90 \%$ of biliary tract cancers (12). Only several effective diagnostic measures and classical symptoms exist, and most patients with GBC are treated at the late stages of the disease, resulting in poor overall prognosis. Lymph node metastasis is the most common form of GBC progression. Given that cancer spread via the lymphatics characterizes the early stages, lymph node metastasis also acts as an important prognostic factor in GBC (13). Thus, identifying a method that can exactly assess lymph node metastasis of GBC before operation bears importance. VEGF-C, an important member of the VEGF family, has been identified as a lymphangiogenic factor. This factor induces formation of lymphatic ducts and promotes lymph node metastasis in combination with VEGF receptor (VEGFR)-3 (14). VEGF-C has also been correlated with lymphatic metastasis in numerous carcinoma cases (7,15-17). Tumor cells secrete high levels of VEGF-C and induce growth of tumor lymphatic vessels. Therefore, sVEGF-C levels may be used as tumor markers to predict lymph node metastasis and prognostic factors for patients with GBC.

To our knowledge, this study was the first to demonstrate that sVEGF-C levels form a positive correlation with VEGF-C expression and LVD and may predict lymph node metastasis and prognosis of patients with GBC. In this study, we used ELISA techniques to analyze preoperative sVEGF-C levels in 51 patients with GBC, 15 patients with chronic cholecystitis, and 10 healthy volunteers. Preoperative sVEGF-C level in patients with GBC was significantly higher than that in healthy volunteers. These discoveries suggest that SVEGF-C level is lower in the normal gallbladder when transitioning into cancerous cells, and tumor cells secrete high levels of VEGF-C, thereby inducing the growth of tumor lymphatic vessels. Our findings agree with those of studies on lung cancer (18), esophageal cancer (19), and colorectal cancer (20). However, no statistical significance was observed in preoperative sVEGF-C levels between patients with GBC and those with chronic cholecystitis. This finding is consistent with other studies indicating that VEGF-C is involved in the formation of inflammatory lymphatic vessels through VEGFR3 in chronic bronchitis (21). Our early research also showed no statistical significance in the expression of VEGF-C between paraffin-embedded tissue specimens of GBC and chronic cholecystitis (7). This finding may imply that VEGF-C is constantly involved in the formation of lymphatic vessels in carcinogenesis of chronic 
Table I. Associations between sVEGF-C, VEGF-C expression, LVD and the clinicopathological characteristics of gallbladder cancer.

\begin{tabular}{|c|c|c|c|c|c|c|c|c|}
\hline \multirow[b]{2}{*}{ Parameters } & \multirow[b]{2}{*}{ Total } & \multirow[b]{2}{*}{ sVEGF-C (pg/ml) } & \multirow[b]{2}{*}{ P-value } & \multicolumn{2}{|c|}{ VEGF-C } & \multirow[b]{2}{*}{ P-value } & \multirow[b]{2}{*}{ LVD (/400 HP) } & \multirow[b]{2}{*}{ P-value } \\
\hline & & & & + & - & & & \\
\hline \multicolumn{9}{|l|}{ Age } \\
\hline$<60$ years & 27 & $7800.8 \pm 1915.1$ & & 20 & 7 & & $7.6 \pm 4.0$ & \\
\hline$\geq 60$ years & 24 & $7897.2 \pm 1956.0$ & 0.86 & 12 & 12 & 0.08 & $6.2 \pm 2.8$ & 0.15 \\
\hline \multicolumn{9}{|l|}{ Sex } \\
\hline Female & 31 & $7934.0 \pm 1921.7$ & & 19 & 12 & & $7.4 \pm 3.7$ & \\
\hline Male & 20 & $7710.0 \pm 1947.7$ & 0.69 & 13 & 7 & 0.79 & $6.2 \pm 3.4$ & 0.27 \\
\hline \multicolumn{9}{|l|}{ Tumor size } \\
\hline$\leq 5 \mathrm{~cm}$ & 40 & $8089.3 \pm 1858.8$ & & 28 & 12 & & $7.0 \pm 3.5$ & \\
\hline$>5 \mathrm{~cm}$ & 11 & $6962.1 \pm 1943.4$ & 0.08 & 4 & 7 & $0.04^{\mathrm{a}}$ & $6.7 \pm 3.8$ & 0.85 \\
\hline \multicolumn{9}{|c|}{ Histological type } \\
\hline Adeno & 23 & $7541.7 \pm 1271.1$ & & 12 & 11 & & $7.0 \pm 3.5$ & \\
\hline Others & 28 & $8096.3 \pm 2309.8$ & 0.31 & 20 & 8 & 0.16 & $6.9 \pm 3.7$ & 0.94 \\
\hline \multicolumn{9}{|c|}{ Histological grading } \\
\hline Poor & 16 & $8035.6 \pm 2084.3$ & & 10 & 6 & & $6.9 \pm 3.6$ & \\
\hline Moderate & 15 & $8231.3 \pm 2594.8$ & & 8 & 7 & & $6.7 \pm 4.2$ & \\
\hline Well & 20 & $7510.8 \pm 1252.4$ & 0.59 & 14 & 6 & 0.6 & $6.9 \pm 3.4$ & 0.31 \\
\hline \multicolumn{9}{|c|}{ Tumor depth } \\
\hline Tis-T2 & 15 & $7999.4 \pm 1913.1$ & & 11 & 4 & & $6.1 \pm 2.6$ & \\
\hline $\mathrm{T} 3-\mathrm{T} 4$ & 36 & $7782.3 \pm 1940.1$ & 0.72 & 21 & 15 & 0.31 & $7.3 \pm 3.9$ & 0.30 \\
\hline \multicolumn{9}{|c|}{ Lymphatic metastasis } \\
\hline Yes & 27 & $8650.0 \pm 1959.9$ & & 21 & 6 & & $8.0 \pm 3.8$ & \\
\hline No & 24 & $6941.8 \pm 1422.3$ & $0.001^{\mathrm{a}}$ & 11 & 13 & $0.02^{\mathrm{a}}$ & $5.7 \pm 2.9$ & $0.02^{\mathrm{a}}$ \\
\hline \multicolumn{9}{|c|}{ Distant metastasis } \\
\hline M0 & 35 & $7860.4 \pm 1694.1$ & & 23 & 12 & & $6.7 \pm 3.3$ & \\
\hline M1 & 16 & $7814.9 \pm 2392.7$ & 0.94 & 9 & 7 & 0.52 & $7.4 \pm 4.1$ & 0.55 \\
\hline \multicolumn{9}{|l|}{ Stage } \\
\hline $0-\mathrm{II}$ & 12 & $7642.3 \pm 1561.5$ & & 9 & 3 & & $6.0 \pm 2.9$ & \\
\hline III-IV & 39 & $7908.9 \pm 2026.2$ & 0.68 & 23 & 16 & 0.32 & $7.2 \pm 3.7$ & 0.33 \\
\hline
\end{tabular}

${ }^{\text {a }}<<0.05$. sVEGF, serum vascular endothelial growth factor; LVD, lymphatic vessel density; HP, high-power field; Adeno, adenocarcinoma.

cholecystitis and easy detection of lymph node metastasis in the early stage of GBC.

This study has analyzed the relationship among sVEGF-C levels, VEGF-C expression, and LVD in GBC. We observed that the positive rate of VEGF-C expression in human GBC tissue with higher sVEGF-C was higher than that with lower sVEGF-C. A statistically significant increase in LVD was noted in higher-sVEGF-C group when compared with the lower-sVEGF-C group. Our results are similar to those of previous research, in which Mathur et al (22) observed that sVEGF-C was related to VEGF-C expression in advanced cervical cancer. These results indicated that sVEGF-C level featured a positive correlation with VEGF-C expression, LVD in GBC, and that the amount of VEGF-C in serum coincided with the expression of VEGF-C in tissue of patients with GBC. We further analyzed the associations among sVEGF-C levels, VEGF-C expression, LVD, and clinicopathological
Table II. sVEGF-C exhibits positive correlation with VEGF-C expression and LVD in gallbladder cancer.

\begin{tabular}{lrrrrr}
\hline & \multicolumn{2}{l}{ VEGF-C } & & LVD & \\
\cline { 2 - 3 }$(\mathrm{pg} / \mathrm{ml})$ & + & - & P-value & $(/ 400 \mathrm{HP})$ & P-value \\
\hline$>7846$ & 25 & 3 & & $8.5 \pm 3.4$ & \\
$\leq 7846$ & 7 & 16 & $<0.001^{\mathrm{a}}$ & $5.0 \pm 2.7$ & $<0.001^{\mathrm{a}}$ \\
\hline
\end{tabular}

${ }^{\text {a }}<<0.01$. sVEGF, serum vascular endothelial growth factor; LVD, lymphatic vessel density.

characteristics of GBC. We observed that the levels of sVEGF-C were related to lymph node metastasis, and that VEGF-C expression in human GBC tissues was significantly correlated 

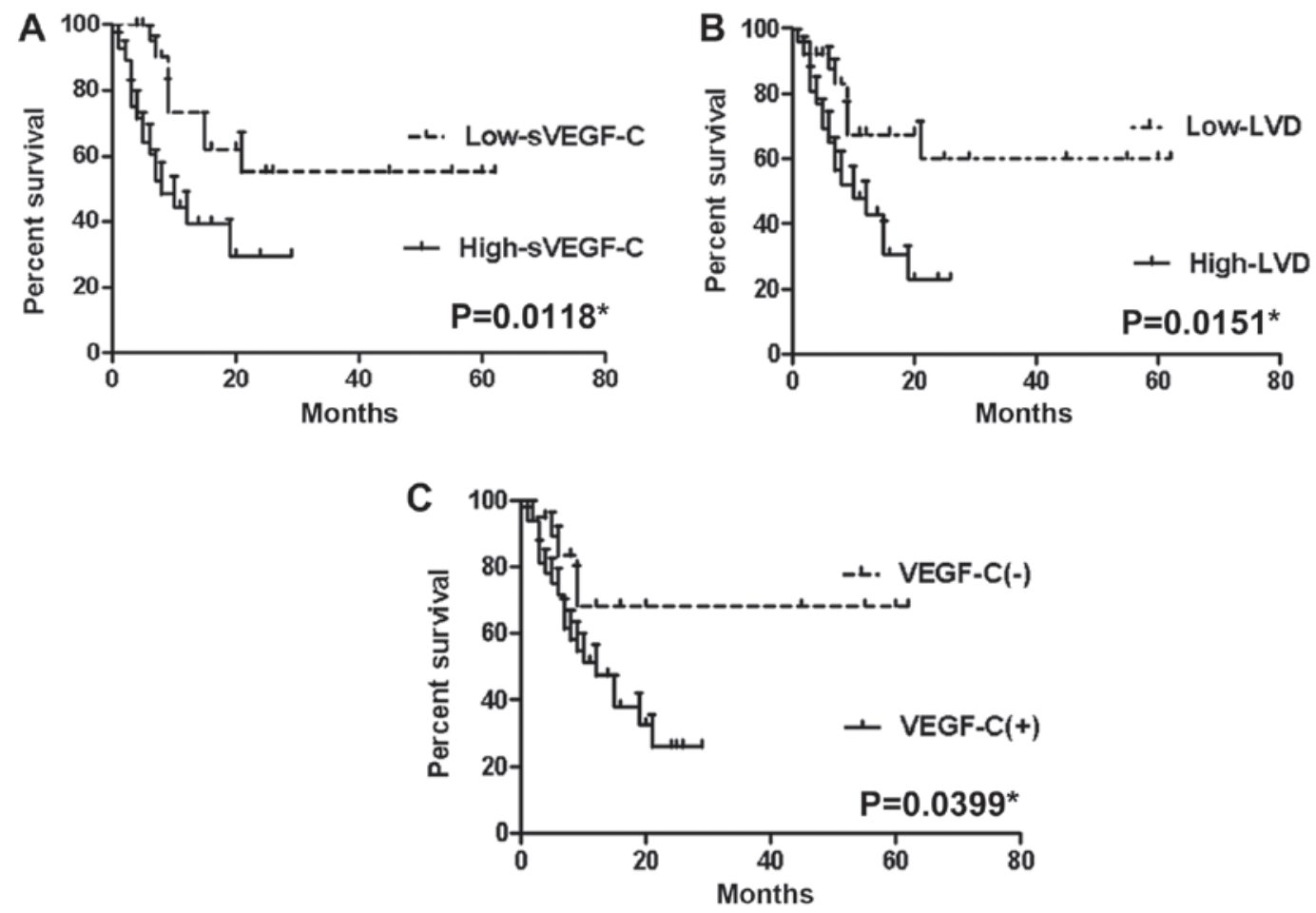

Figure 3. (A) Kaplan-Meier survival curves in the low sVEGF-C ( $<7846 \mathrm{pg} / \mathrm{ml})$ and high sVEGF-C groups ( $>7846 \mathrm{pg} / \mathrm{ml})$. (B) Kaplan-Meier survival curves in the high ( $\geq 7 / 400 \mathrm{HP}$ ) and low ( $<7 / 400 \mathrm{HP}$ ) LVD groups. (C) Kaplan-Meier survival curves in the VEGF-C-positive and negative expression groups. VEGF-C, vascular endothelial growth factor-C; sVEGF, serum vascular endothelial growth factor; LVD, lymphatic vessel density; HP, high-power field.

with lymph node metastasis and tumor size. LVD in human GBC tissues was also significantly correlated with lymph node metastasis. These findings are consistent with those of other studies (18), which revealed that sVEGF-C level was higher in patients with non-small-cell lung cancer with lymph node metastasis than in those without lymph node metastasis. These results suggest that $\mathrm{SVEGF-C} \mathrm{levels} \mathrm{may} \mathrm{predict} \mathrm{lymph} \mathrm{node}$ metastasis of patients with GBC.

Various studies showed that SVEGF-C levels can predict tumor prognosis. Gisterek (23) et al observed that high sVEGF-C level was related to poor prognosis in patients with breast tumors. Wang (24) et al have shown the association of sVEGF-C and prognosis of patients with gastric cancer. In this study, mean survival time of patients with high sVEGF-C level was significantly shorter than that with low sVEGF-C level. We have further analyzed that mean survival time was shorter in the VEGF-C-positive expression group than in the negative expression group. Mean survival time of patients with high LVD was also significantly shorter than that with low LVD. These studies suggested that sVEGF-C, VEGF-C, and LVD can be useful in predicting prognosis of patients with GBC as high sVEGF-C levels in patients with increases LVD, thereby promoting lymph node metastasis and resulting in poor outcomes.

We concluded that a preoperative high-sVEGF-C level is related to VEGF-C expression in human GBC tissues, LVD, and lymph node metastasis. These findings suggest that preoperative sVEGF-C level serves as a useful biomarker for assessment of the need for anti-lymphatic metastasis therapy. We also deduced that preoperative VEGF-C level may reflect malignancies, such as lymph node metastasis, and may predict prognosis in patients with GBC.

\section{Acknowledgements}

Not applicable.

\section{Funding}

The present study was funded by the Youth Foundation of Fujian Provincial Health and Family Planning Commission (grant no. 2014-1-49), Fujian Provincial Scientific and Technological Innovation Joint Fund Project (grant no. 2017Y9029) and The National Natural Science Foundation (grant no. 81672468) in China.

\section{Availability of data and materials}

All data generated or analyzed during this study are included in this published article.

\section{Authors' contributions}

LJ and LX conceived and designed the study. LJ, ML, XC, LX, FS and YC assisted with the collection of the data. LJ, ML, $\mathrm{XC}, \mathrm{LX}$ and FS performed the data analysis and interpretation of the results. LJ wrote the manuscript. All authors approved the final version of the manuscript for publication.

\section{Ethics approval and consent to participate}

All procedures performed in the study involving human participants were in accordance with the protocol approved by the Ethics Committee of the Affiliated Union Hospital of Fujian Medical University. 


\section{Patient consent for publication}

Not applicable.

\section{Competing interests}

The authors declare that they have no competing interests.

\section{References}

1. Muratore A, Polastri R and Capussotti L: Radical surgery for gallbladder cancer: Current options. Eur J Surg Oncol 26 : 438-443, 2000

2. Schoppmann SF, Fenzl A, Nagy K, Unger S, Bayer G, Geleff S, Gnant M, Horvat R, Jakesz R and Birner P: VEGF-C expressing tumor-associated macrophages in lymph node positive breast cancer: Impact on lymphangiogenesis and survival. Surgery 139 : 839-846, 2006

3. Mattila MM, Ruohola JK, Karpanen T, Jackson DG, Alitalo K and Härkönen PL: VEGF-C induced lymphangiogenesis is associated with lymph node metastasis in orthotopic MCF-7 tumors. Int J Cancer 98: 946-951, 2002.

4. Tanaka T, Ishiguro H, Kuwabara Y, Kimura M, Mitsui A, Katada T, Shiozaki M, Naganawa Y, Fujii Y and Takeyama H: Vascular endothelial growth factor C (VEGF-C) in esophagea cancer correlates with lymph node metastasis and poor patient prognosis. J Exp Clin Cancer Res 29: 83, 2010.

5. Karpanen T, Egeblad M, Karkkainen MJ, Kubo H Ylä-Herttuala S, Jäättelä M and Alitalo K: Vascular endothelial growth factor $\mathrm{C}$ promotes tumor lymphangiogenesis and intralymphatic tumor growth. Cancer Res 61: 1786-1790, 2001.

6. Kitadai Y, Amioka T, Haruma K, Tanaka S, Yoshihara M, Sumii K Matsutani N, Yasui W and Chayama K: Clinicopathological significance of vascular endothelial growth factor (VEGF)-C in human esophageal squamous cell carcinomas. Int J Cancer 93: 662-666, 2001

7. Jiang L, Chen YL, She FF, Tang NH, Li XJ and Wang XX: Expressions of VEGF-C and VEGF-D and their correlation with lymphangiogenesis and angiogenesis in gallbladder carcinoma. Zhonghua Zhong Liu Za Zhi 32: 190-195, 2010 (In Chinese).

8. Edge SB and Compton CC: The American Joint Committee on Cancer: The 7th edition of the AJCC cancer staging manual and the future of TNM. Ann Surg Oncol 17: 1471-1474, 2010

9. Chen Y, Jiang L, She F, Tang N, Wang X, Li X, Han S and Zhu J: Vascular endothelial growth factor-C promotes the growth and invasion of gallbladder cancer via an autocrine mechanism. Mol Cell Biochem 345: 77-89, 2010.

10. Takanami I: Lymphatic microvessel density using D2-40 is associated with nodal metastasis in non-small cell lung cancer. Oncol Rep 15: 437-442, 2006.

11. Varshney S, Butturini G and Gupta R: Incidental carcinoma of the gallbladder. Eur J Surg Oncol 28: 4-10, 2002.

12. Lazcano-Ponce EC, Miquel JF, Muñoz N, Herrero R, Ferrecio C, Wistuba II, Alonso de Ruiz P, Aristi Urista G and Nervi F: Epidemiology and molecular pathology of gallbladder cancer. CA Cancer J Clin 51: 349-364, 2001.
13. Shimada H, Endo I, Togo S, Nakano A, Izumi $\mathrm{T}$ and Nakagawara G: The role of lymph node dissection in the treatment of gallbladder carcinoma. Cancer 79: 892-899, 1997.

14. Joukov V, Pajusola K, Kaipainen A, Chilov D, Lahtinen I, Kukk E, Saksela O, Kalkkinen N and Alitalo K: A novel vascular endothelial growth factor, VEGF-C, is a ligand for the Flt4 (VEGFR-3) and KDR (VEGFR-2) receptor tyrosine kinases. EMBO J 15: 290-298, 1996.

15. Ito Y, Shibata MA, Eid N, Morimoto J and Otsuki Y: Lymphangiogenesis and axillary lymph node metastases correlated with VEGF-C expression in two immunocompetent mouse mammary carcinoma models. Int J Breast Cancer 2011: 867152, 2011.

16. Maula SM, Luukkaa M, Grénman R, Jackson D, Jalkanen S and Ristamäki R: Intratumoral lymphatics are essential for the metastatic spread and prognosis in squamous cell carcinomas of the head and neck region. Cancer Res 63: 1920-1926, 2003.

17. Oh SJ, Jeltsch MM, Birkenhäger R, McCarthy JE, Weich HA, Christ B, Alitalo K and Wilting J: VEGF and VEGF-C: Specific induction of angiogenesis and lymphangiogenesis in the differentiated avian chorioallantoic membrane. Dev Biol 188 96-109, 1997.

18. Tamura M and Ohta Y: Serum vascular endothelial growth factor-C level in patients with primary nonsmall cell lung carcinoma: A possible diagnostic tool for lymph node metastasis. Cancer 98: 1217-1222, 2003.

19. Kozlowski M, Kowalczuk O, Milewski R, Chyczewski L, Niklinski J and Laudański J: Serum vascular endothelial growth factors $\mathrm{C}$ and $\mathrm{D}$ in patients with oesophageal cancer. Eur J Cardiothorac Surg 38: 260-267, 2010.

20. $\mathrm{Xu} \mathrm{T}$ and Chen D: Serum vascular endothelial growth factor- $\mathrm{C}$ and vascular endothelial growth factor level in patients with colorectal carcinoma and clinical significance. J Huazhong Univ Sci Technolog Med Sci 26: pp329-331, 2006.

21. Baluk P, Tammela T, Ator E, Lyubynska N, Achen MG, Hicklin DJ, Jeltsch M, Petrova TV, Pytowski B, Stacker SA, et al: Pathogenesis of persistent lymphatic vessel hyperplasia in chronic airway inflammation. J Clin Invest 115: 247-257, 2005.

22. Mathur SP, Mathur RS, Gray EA, Lane D, Underwood PG, Kohler M and Creasman WT: Serum vascular endothelial growth factor C (VEGF-C) as a specific biomarker for advanced cervical cancer: Relationship to insulin-like growth factor II (IGF-II), IGF binding protein 3 (IGF-BP3) and VEGF-A [corrected]. Gynecol Oncol 98: 467-483, 2005.

23. Gisterek I, Matkowski R, Lacko A, Sedlaczek P, Szewczyk K, Biecek P, Halon A, Staszek U, Szelachowska J, Pudelko M, et al: Serum vascular endothelial growth factors a, $\mathrm{C}$ and $\mathrm{d}$ in human breast tumors. Pathol Oncol Res 16: 337-344, 2010.

24. Wang TB, Deng MH, Qiu WS and Dong WG: Association of serum vascular endothelial growth factor-C and lymphatic vessel density with lymph node metastasis and prognosis of patients with gastric cancer. World J Gastroenterol 13: 1794-1798, 2007. 